Proyecciones Journal of Mathematics

Vol. 33, Nº 3, pp. 325-347, September 2014.

Universidad Católica del Norte

Antofagasta - Chile

\title{
On the qualitative properties of differential equations of third order with retarded argument
}

\author{
Cemil Tunç \\ Yüzüncü Yil University, Turkey \\ and \\ Sizar Abid Mohammed \\ University of Duhok, Iraq \\ Received : April 2014. Accepted : June 2014
}

\begin{abstract}
By using the standard Liapunov-Krasovskii functional approach, in this paper, new stability, boundedness and ultimately boundedness criteria are established for a class of vector functional differential equations of third order with retarded argument.
\end{abstract}




\section{Introduction}

Differential equations with retarded argument are used to describe many phenomena of physical interest. While ordinary differential equations without delay contain derivatives which depend on the solution at the present value of the independent variable ('time', differential equations with retarded argument contain in addition derivatives which depend on the solution at previous times. Differential equations with retarded argument arise in models throughout the sciences. Systems of differential equations with retarded argument now occupy a place of central importance in all areas of science and particularly in the biological sciences (e.g., population dynamics and epidemiology). Interest in such systems often arises when traditional point wise modeling assumptions are replaced by more realistic distributed assumptions, for example, when the birth rate of predators is affected by prior levels of predators or prey rather than by only the current levels in a predator-prey model. The manner in which the properties of systems of differential equations with retarded argument differ from those of systems of ordinary differential equations has been and remains an active area of research (see, e.g. Ahmad and Rama Mohana Rao [4], Burton [5], Èl'sgol'ts and Norkin [11], Hale [15], Hara [16], Krasovskii [17], Rauch [24], Smith [26] and references therein). On the other hand, differential equations of third order have been proved to be valuable tools in the modeling of many phenomena in various fields of science and engineering.Indeed, we can find some applications such as nonlinear oscillations (Afuwape et al. [3], Fridedrichs [14]), prototypical examples of complex dynamical systems in a high-dimensional phase space, displacement in a mechanical system, velocity, acceleration (Chlouverakis and Sprott [6], Eichhorn et al. [10], Linz [19]), the biological model and other models (Cronin- Scanlon [8]), electronic theory (Rauch [24]), the boundedness of some minimal chaotic attractors (Elhadj and Sprott [9]) and etc. Therefore, it is worth to investigate the qualitative behavior of differential equations of third order. At the same time, the theory of stability and boundedness of solutions are important branches of the qualitative theory of differential equations. In the last 60 years, there has been increasing interest in obtaining the sufficient conditions for the stability/instability/boundedness/ ultimately boundedness etc. of solutions of different classes of differential equations of third order such as linear and nonlinear ordinary, and functional differential equations; for a comprehensive treatment of subject, we refer the reader to the book by Reissig et al. [25] as a survey and the papers by Ademola and Arawomo [1], 
Afuwape and Castellanos [2], Afuwape et. al. [3], Chukwu [7], Ezeilo ([12], [13]), Hara [16], Mehri and Shadman [20], Meng [21], Omeike and Afuwape [22], Qian [23], Rauch [24], Smith [26], Swick [27], Tejumola [28], Tunc [2940], Tunc and Ates [41], Tunc and Ergören [42], Wall and Moe [43], Zhang and $\mathrm{Yu}[44]$ and references therein). It is well known that an important tool in the study of stability/instability/boundedness/ ultimately boundedness of solutions of the considerable number of ordinary/neutral/retarded differential equations of higher order is the Liapunov function/LiapunovKrasovskii functional techniques. The Liapunov function technique goes back for ordinary differential equations as far as the approximation methods of Liapunov (1857-1918) (see, also, Liapunov [18]), which he developed in 1899 , make it possible to define the stability of sets of ordinary differential equations and he created the modern theory of the stability of a dynamic system. Besides, in 1959, Krasovskii (1924-2012) carried out LiapunovKrasovskii functional technique for a treatment of global behavior of solutions of a class of nonlinear differential equations with delay by suitable Liapunov- Krasovskii functionals. In his work, Krasovskii indicated the extension of Liapunov's method to a class of general differential equations with time delay in the literature. Especially, after the work of Krasovskii [17], stability/instability/boundedness/ convergence/ existence of periodic solutions, etc. of solutions of scalar neutral/retarded differential equations of higher order were investigated quite extensively by many authors (see the mentioned references and the references therein). It should be noted that, today, the same topics are also being investigated intensely in the literature and many good results are being obtained by the researchers. We would not like to give the details.

In 1968, Wall and Moe [43] considered the scalar differential equation of third order without delay

$$
x^{\prime \prime}+\left(1+x^{\prime 2}\right) x^{\prime}+x^{\prime}+x=0 .
$$

The authors proved that the functional relationship to the original equations is evident by the direct way in which the desired Liapunov function was obtained from the original differential equations, and they gave sufficient conditions which quarantee globally asymptotic stability of this equation.

Later, in 2009, the result of Wall and Moe [43] was improved and extended by Tunc [35] for the stability and boundedness of the following 
vector differential equation of third order without delay:

$$
X^{\prime \prime}+\Psi\left(X^{\prime}\right) X^{\prime \prime}+B X^{\prime}+c X=P(t)
$$

when $P(t) \equiv 0$ and $P(t)=0$, respectively.

After that, in 2010, Omeike and Afuwape [22] studied the ultimate boundedness of solutions of Eq. (3). By this work, they did a good contribution to the topic. To the best of our knowledge from the literature, by now, no attention was given to the investigation of the stability/boundedness/ ultimately boundedness of solutions of the vector functional differential equations of third order with retardation. The basic reason for the absence of any work on this topic may be the difficulty of the construction or definition of appropriate Liapunov- Krasovskii functionals for differential equations of third order with retardation. Further, until now, construction or definition of suitable Liapunov functions/Liapunov- Krasovskii functionals for higher order nonlinear differential equations without and with retardation remains an as open problem in the literature. Therefore, it is worthwhile to do the discussion of the mentioned properties for those equations.

In this paper, we are concerned with the stability, boundedness and ultimately boundedness properties of the following type vector functional differential equations of third order with retarded argument, $\tau_{1}>0$ :

$$
X^{\prime \prime \prime}+\Psi\left(X^{\prime}\right) X^{\prime \prime}+B X^{\prime}\left(t-\tau_{1}\right)+c X\left(t-\tau_{1}\right)=P(t),
$$

where $\tau_{1}$ is the retarded argument; $c$ is a positive constant; $B$ is $n \times n$ an constant symmetric matrix; $\Psi$ is an $n \times n$ - continuous differentiable symmetric matrix function such that the Jacobian matrix $J\left(\Psi\left(X^{\prime}\right) X^{\prime} \mid X^{\prime}\right)$ exists and is symmetric and continuous, that is,

$$
\begin{aligned}
& \mathrm{J}\left(\psi\left(X^{\prime}\right) X^{\prime} \mid X^{\prime}\right)=\frac{\partial}{\partial x_{j}^{\prime}}\left(\sum_{k=1}^{n} \psi_{i k} x^{\prime}{ }_{k}\right) \\
& =\psi\left(X^{\prime}\right)+\sum_{k=1}^{n} \frac{\partial \psi_{i k}}{\partial x^{\prime}{ }_{j}} x_{k}^{\prime}, \quad(i, j=1,2, \ldots, n),
\end{aligned}
$$

exists and is symmetric and continuous, where $\left(x^{\prime}{ }_{1}, x^{\prime}{ }_{2}, \ldots, x_{n}^{\prime}\right)$ and $\left(\psi_{i k}\right)$ are components of $X^{\prime}$ and $\Psi$, respectively; $P: \Re^{+} \rightarrow \Re^{n}$ is a continuous function, $\Re^{+}=[0, \infty)$ and the primes in Eq.(1) indicate differentiation with respect to $t, t \geq t_{0} \geq 0$. For the third order functional differential equation with the retarded argument, let $X=X_{1}, X^{\prime}=X_{2}, X^{\prime \prime}=X_{3}$, then Eq. (1) can be written in the form

$$
X^{\prime}{ }_{1}=X_{2},
$$




$$
\begin{gathered}
X^{\prime}{ }_{2}=X_{3}, \\
X_{3}^{\prime}=-\Psi\left(X_{2}\right) X_{3}-B X_{2}+\int_{t-\tau_{1}}^{t} B X_{3}(s) d s \\
-c X_{1}+c \int_{t-\tau_{1}}^{t} X_{2}(s) d s+P(t) .
\end{gathered}
$$

Throughout this paper, we assume that the existence and the uniqueness of the solutions of Eq. (1) hold ( $\mathrm{El}^{\prime} \mathrm{sgol}^{\prime}$ ts and Norkin [11]. The motivation of this paper comes from the results established in Wall and Moe [43], Tunç [35], Omeike and Afuwape [22] and mentioned papers. The main purpose of this paper is to get some new stability/boundedness/ ultimately boundedness results of Eq. (1) by using the Liapunov- Krasovskii functional approach. This paper is the first attempt in the literature to obtain sufficient conditions which guarantee the stability/boundedness/ultimately boundedness of vector functional differential equations of third order with retarded argument, and it has a new contribution the topic in the literature. This case shows the novelty of this work. It should also be noted that the results to be established here may be useful for researchers working on the qualitative behaviors of solutions.

One tool to be used here is LaSalle's invariance principle. If we consider delay differential system

$$
\dot{x}=F\left(x_{t}\right), x_{t}=x(t+\theta),-r \leq \theta \leq 0, t \geq 0,
$$

we take $C=C\left([-r, 0], \Re^{n}\right)$ to be the space of continuous function from $[-r, 0]$ into $\Re^{n}$ and ask that $F: C \rightarrow \Re^{n}$ be continuous. We say that $V: C \rightarrow \Re$ is a Liapunov function on a set $G \subset C$ relative to $F$ if $V$ is continuous on $\bar{G}$, the closure of $G, \dot{V}$ is defined on $G$, and $\dot{V} \leq 0$ on $G$.

The following form of LaSalle's invariance principle can be found in Smith [26, Theorem 5.17]. Here, $\omega$ denotes the omega limit set of a solution.

Theorem A. If $V$ is a Liapunov function on $G$ and $x_{t}(\phi)$ is a bounded solution such that $x_{t}(\phi) \in G$ for $t \geq 0$, then $\omega(\phi) \neq 0$ is contained in the largest invariant subset of $E \equiv\{\psi \in \bar{G}: \dot{V}(\psi)=0\}$. 


\section{Main results}

Before we state our main results, we give an algebraic result which will be required in the proofs.

Lemma. Let $A$ be a real symmetric $n \times n$-matrix. Then for any $X_{1} \in R^{n}$

$$
\delta_{a}\left\|X_{1}\right\|^{2} \leq\left\langle A X_{1}, X_{1}\right\rangle \leq \Delta_{a}\left\|X_{1}\right\|^{2},
$$

where $\delta_{a}$ and $\Delta_{a}$ are, respectively, the least and greatest eigenvalues of the matrix $A$ (see [22]).

Let $P(t) \equiv 0$. The first main result of this paper is the following theorem.

Theorem 1. In addition to the basic assumptions imposed on $\Psi\left(X_{2}\right)$, $B$ and $c$, we assume that $P(t) \equiv 0$ and there exist positive constants $\alpha, \varepsilon, a_{0}, a_{1}, b_{0}$ and $c$ such that the following conditions hold:

$n \times n$ - symmetric matrices $B$ and $\Psi$ commute with each other and

$$
a_{0} b_{0}-c>0,1-\alpha a_{0}>0, b_{0} \leq \lambda_{i}(B) \leq b_{1}
$$

and

$$
a_{0}+\varepsilon \leq \lambda_{i}\left(\Psi\left(X_{2}\right)\right) \leq a_{1} \text { for all } X_{2} \in \Re^{n} .
$$

if

$$
\begin{gathered}
\tau_{1}<\min \left\{\frac{\alpha a_{0} b_{0} c}{\alpha a_{0} b_{0} b_{1}+\alpha a_{0} b_{0} c}, \frac{k_{1}}{\left(2 a_{0}+\alpha a_{0} b_{0}+1\right) c+a_{0} b_{1}},\right. \\
\left.\frac{k_{2}}{c+\left(2+a_{0}+\alpha a_{0} b_{0}\right) b_{1}}\right\}
\end{gathered}
$$

then, all solutions of Eq. (1) are bounded and the zero solution of Eq. (1) is globally asymptotically stable, where

$$
k_{1}=2\left(a_{0} b_{0}-c\right)-\alpha a_{0} b_{0}\left[a_{0}+c^{-1}\left(b_{1}-b_{0}\right)^{2}\right]>0
$$

and

$$
k_{2}=2 \varepsilon\left[1-\alpha a_{0} b_{0} c^{-1}\left(a_{1}-a_{0}\right)^{2}\right]>0 .
$$

Proof. To prove the theorem, we define a differentiable Liapunov- Krasovskii functional $V(t)=V\left(X_{1}(t), X_{2}(t), X_{3}(t)\right)$ by 


$$
\begin{gathered}
2 V=a_{0} c\left\langle X_{1}, X_{1}\right\rangle+a_{0} \int_{0}^{1}\left\langle\sigma \Psi\left(\sigma X_{2}\right) X_{2}, X_{2}\right\rangle d \sigma+\alpha a_{0} b_{0}^{2}\left\langle X_{1}, X_{1}\right\rangle \\
+\left\langle B X_{2}, X_{2}\right\rangle+\left\langle X_{3}, X_{3}\right\rangle+2 \alpha a_{0}^{2} b_{0}\left\langle X_{1}, X_{2}\right\rangle+2 \alpha a_{0} b_{0}\left\langle X_{1}, X_{3}\right\rangle \\
+2 a_{0}\left\langle X_{2}, X_{3}\right\rangle+2 c\left\langle X_{1}, X_{2}\right\rangle-\alpha a_{0} b_{0}\left\langle X_{2}, X_{2}\right\rangle \\
+2 \lambda_{1} \int_{-\tau_{1}}^{0} \int_{t+s}^{t}\left\|X_{2}(\theta)\right\|^{2} d \theta d s+2 \eta_{1} \int_{-\tau_{1}}^{0} \int_{t+s}^{t}\left\|X_{3}(\theta)\right\|^{2} d \theta d s
\end{gathered}
$$

where

$$
0<\alpha<\min \left\{\frac{1}{a_{0}}, \frac{a_{0}}{b_{0}}, \frac{a_{0} b_{0}-c}{a_{0} b_{0}\left[a_{0}+c^{-1}\left(b_{1}-b_{0}\right)^{2}\right]}, \frac{c}{a_{0} b_{0}\left(a_{1}-a_{0}\right)}\right\}
$$

$a_{1}>a_{0}, b_{1} \neq b_{0}$ and $\lambda_{1}$ and $\eta_{1}$ are certain positive constants to be determined later in the proof.

From (4), it follows that

$$
\begin{gathered}
2 V=a_{0} b_{0}\left\|a_{0}{ }^{-\frac{1}{2}} X_{2}+a_{0}{ }^{-\frac{1}{2}} b_{0}{ }^{-1} c X_{1}\right\|^{2}+\left\|X_{3}+a_{0} X_{2}+\alpha a_{0} b_{0} X_{1}\right\|^{2} \\
+a_{0} \int_{0}^{1}\left\langle\sigma \Psi\left(\sigma X_{2}\right) X_{2}, X_{2}\right\rangle d \sigma-2 a_{0}^{2}\left\langle X_{2}, X_{2}\right\rangle+\left\langle\left(B-b_{0} I\right) X_{2}, X_{2}\right\rangle \\
+\alpha a_{0} b_{0}^{2}\left(1-\alpha a_{0}\right)\left\langle X_{1}, X_{1}\right\rangle+c\left(a_{0}-c b_{0}^{-1}\right)\left\langle X_{1}, X_{1}\right\rangle+a_{0}\left(a_{0}-\alpha b_{0}\right)\left\langle X_{2}, X_{2}\right\rangle \\
+2 \lambda_{1} \int_{-\tau_{1}}^{0} \int_{t+s}^{t}\left\|X_{2}(\theta)\right\|^{2} d \theta d s+2 \eta_{1} \int_{-\tau_{1}}^{0} \int_{t+s}^{t}\left\|X_{3}(\theta)\right\|^{2} d \theta d s .
\end{gathered}
$$

In view of the assumptions of Theorem 1 , it is clear that

$$
\begin{gathered}
V(0,0,0)=0, \\
a_{0} \int_{0}^{1}\left\langle\sigma \Psi\left(\sigma X_{2}\right) X_{2}, X_{2}\right\rangle d \sigma-2 a_{0}^{2}\left\langle X_{2}, X_{2}\right\rangle \\
=a_{0} \int_{0}^{1}\left\langle\left(\sigma \Psi\left(\sigma X_{2}\right)-a_{0} I\right) X_{2}, X_{2}\right\rangle d \sigma \geq \varepsilon a_{0}\left\|X_{2}\right\|^{2},
\end{gathered}
$$




$$
\begin{gathered}
\left\langle\left(B-b_{0} I\right) X_{2}, X_{2} \geq 0,\right. \\
\alpha a_{0} b_{0}^{2}\left(1-\alpha a_{0}\right)\left\langle X_{1}, X_{1}\right\rangle=\mu_{1}\left\|X_{1}\right\|^{2}, \\
\mu_{1}=\alpha a_{0} b_{0}^{2}\left(1-\alpha a_{0}\right)>0, \\
c\left(a_{0}-c b_{0}^{-1}\right)\left\langle X_{1}, X_{1}\right\rangle=\mu_{2}\left\|X_{1}\right\|^{2}, \\
\mu_{2}=c\left(a_{0}-c b_{0}^{-1}\right)>0, \\
a_{0}\left(a_{0}-\alpha b_{0}\right)\left\langle X_{2}, X_{2}\right\rangle=\mu_{3}\left\|X_{2}\right\|^{2}, \\
\mu_{3}=a_{0}\left(a_{0}-\alpha b_{0}\right)>0 .
\end{gathered}
$$

By noting the above discussion, we get

$$
\begin{aligned}
& V \geq \frac{1}{2} a_{0} b_{0}\left\|a_{0}^{-\frac{1}{2}} X_{2}+a_{0}^{-\frac{1}{2}} b_{0}^{-1} c X_{1}\right\|^{2}+\frac{1}{2}\left\|X_{3}+a_{0} X_{2}+\alpha a_{0} b_{0} X_{1}\right\|^{2} \\
& +\frac{1}{2}\left(\mu_{1}+\mu_{2}\right)\left\|X_{1}\right\|^{2}+\frac{1}{2}\left(a_{0} \varepsilon+\mu_{3}\right)\left\|X_{2}\right\|^{2} \\
& +2 \lambda_{1} \int_{-\tau_{1}}^{0} \int_{t+s}^{t}\left\|X_{2}(\theta)\right\|^{2} d \theta d s+2 \eta_{1} \int_{-\tau_{1}}^{0} \int_{t+s}^{t}\left\|X_{3}(\theta)\right\|^{2} d \theta d s .
\end{aligned}
$$

Thus, one can obtain from the above estimate that there exist sufficiently small positive constants $d_{i},(i=1,2,3)$, such that

$$
V \geq d_{1}\left\|X_{1}\right\|^{2}+d_{2}\left\|X_{2}\right\|^{2}+d_{3}\left\|X_{3}\right\|^{2} .
$$

Then, we conclude that Liapunov-Krasovskii functional $V$ is positive definite. Let

$$
d_{4}=\min \left\{d_{1}, d_{2}, d_{3}\right\}
$$

Then, it is clear that

$$
V \geq d_{4}\left(\left\|X_{1}\right\|^{2}+\left\|X_{2}\right\|^{2}+\left\|X_{3}\right\|^{2}\right) .
$$

$\operatorname{Let}\left(X_{1}, X_{2}, X_{3}\right)=\left(X_{1}(t), X_{2}(t), X_{3}(t)\right)$ be any solution of system (2). Differentiating this functional, (3), with respect to along system (2), we have 


$$
\begin{aligned}
& \dot{V}=-\alpha a_{0} b_{0} c\left\langle X_{1}, X_{1}\right\rangle-a_{0}\left\langle X_{2}, B X_{2}\right\rangle+c\left\langle X_{2}, X_{2}\right\rangle \\
& +\alpha a_{0}^{2} b_{0}\left\langle X_{2}, X_{2}\right\rangle-\alpha a_{0} b_{0}\left\langle X_{1}, \Psi\left(X_{2}\right) X_{3}\right\rangle \\
& +\alpha a_{0}^{2} b_{0}\left\langle X_{1}, X_{3}\right\rangle-\left\langle\Psi\left(X_{2}\right) X_{3}, X_{3}\right\rangle \\
& +a_{0}\left\langle X_{3}, X_{3}\right\rangle-\alpha a_{0} b_{0}\left\langle X_{1}, B X_{2}\right\rangle \\
& +\alpha a_{0} b_{0}^{2}\left\langle X_{1}, X_{2}\right\rangle+\left\langle X_{3}, \int_{t-\tau_{1}}^{t} B X_{3}(s) d s\right\rangle \\
& +\left\langle X_{3}, c \int_{t-\tau_{1}}^{t} X_{2}(s) d s\right\rangle+\alpha a_{0} b_{0}\left\langle X_{1}, \int_{t-\tau_{1}}^{t} B X_{3}(s) d s\right\rangle \\
& +\alpha a_{0} b_{0} c\left\langle X_{1}, \int_{t-\tau_{1}}^{t} X_{2}(s) d s\right\rangle+a_{0}\left\langle X_{2}, \int_{t-\tau_{1}}^{t} B X_{3}(s) d s\right\rangle \\
& +a_{0} c\left\langle X_{2}, \int_{t-\tau_{1}}^{t} X_{2}(s) d s\right\rangle+\lambda_{1} \tau_{1}\left\langle X_{2}, X_{2}\right\rangle+\eta_{1} \tau_{1}\left\langle X_{3}, X_{3}\right\rangle \\
& -\lambda_{1} \int_{t-\tau_{1}}^{t}\left\|X_{2}(s)\right\|^{2} d s-\eta_{1} \int_{t-\tau_{1}}^{t}\left\|X_{3}(s)\right\|^{2} d s \\
& =-\frac{1}{2} \alpha a_{0} b_{0} c\left\langle X_{1}, X_{1}\right\rangle-\left\langle\left(a_{0} B-c I-\alpha a_{0}^{2} b_{0} I\right) X_{2}, X_{2}\right\rangle \\
& -\left\langle\left(\Psi\left(X_{2}\right)-a_{0} I\right) X_{3}, X_{3}\right\rangle \\
& -\frac{1}{4} \alpha a_{0} b_{0}\left\|c^{\frac{1}{2}} X_{1}+2 c^{-\frac{1}{2}}\left(\Psi\left(X_{2}\right)-a_{0} I\right) X_{3}\right\|^{2} \\
& +\frac{1}{4} \alpha a_{0} b_{0}\left\|2 c^{-\frac{1}{2}}\left(\Psi\left(X_{2}\right)-a_{0} I\right) X_{3}\right\|^{2} \\
& -\frac{1}{4} \alpha a_{0} b_{0}\left\|c^{\frac{1}{2}} X_{1}+2 c^{-\frac{1}{2}}\left(B-b_{0} I\right) X_{2}\right\|^{2} \\
& +\frac{1}{4} \alpha a_{0} b_{0}\left\|2 c^{-\frac{1}{2}}\left(B-b_{0} I\right) X_{2}\right\|^{2} \\
& +\left\langle X_{3}, \int_{t-\tau_{1}}^{t} B X_{3}(s) d s\right\rangle+\left\langle X_{3}, c \int_{t-\tau_{1}}^{t} X_{2}(s) d s\right\rangle \\
& +\alpha a_{0} b_{0}\left\langle X_{1}, \int_{t-\tau_{1}}^{t} B X_{3}(s) d s\right\rangle+\alpha a_{0} b_{0} c\left\langle X_{1}, \int_{t-\tau_{1}}^{t} X_{2}(s) d s\right\rangle \\
& +a_{0}\left\langle X_{2}, \int_{t-\tau_{1}}^{t} B X_{3}(s) d s\right\rangle+a_{0} c\left\langle X_{2}, \int_{t-\tau_{1}}^{t} X_{2}(s) d s\right\rangle \\
& +\lambda_{1} \tau_{1}\left\langle X_{2}, X_{2}\right\rangle+\eta_{1} \tau_{1}\left\langle X_{3}, X_{3}\right\rangle
\end{aligned}
$$


$-\lambda_{1} \int_{t-\tau_{1}}^{t}\left\|X_{2}(s)\right\|^{2} d s-\eta_{1} \int_{t-\tau_{1}}^{t}\left\|X_{3}(s)\right\|^{2} d s$.

Using the assumptions of Theorem 1 and elementary estimates, we obtain

$$
\begin{aligned}
& \left\langle X_{3}, \int_{t-\tau_{1}}^{t} B X_{3}(s) d s \leq\left\|X_{3}\right\|\left\|\int_{t-\tau_{1}}^{t} B X_{3}(s)\right\| d s\right. \\
& \leq b_{1}\left\|X_{3}\right\| \int_{t-\tau_{1}}^{t}\left\|X_{3}(s)\right\| d s \\
& \leq \frac{1}{2} b_{1} \int_{t-\tau_{1}}^{t}\left\{\left\|X_{3}(t)\right\|^{2}+\left\|X_{3}(s)\right\|^{2}\right\} d s \\
& =\frac{1}{2} b_{1} \tau_{1}\left\|X_{3}\right\|^{2}+\frac{1}{2} b_{1} \int_{t-\tau_{1}}^{t}\left\|X_{3}(s)\right\|^{2} d s, \\
& \left\langle X_{3}, c \int_{t-\tau_{1}}^{t} X_{2}(s) d s\right\rangle \leq c\left\|X_{3}\right\|\left\|\int_{t-\tau_{1}}^{t} X_{2}(s) d s\right\| \\
& \leq c\left\|X_{3}\right\| \int_{t-\tau_{1}}^{t}\left\|X_{2}(s)\right\| d s \\
& \leq \frac{1}{2} c \int_{t-\tau_{1}}^{t}\left\{\left\|X_{3}(t)\right\|^{2}+\left\|X_{2}(s)\right\|^{2}\right\} d s \\
& =\frac{1}{2} c \tau_{1}\left\|X_{3}\right\|^{2}+\frac{1}{2} c \int_{t-\tau_{1}}^{t}\left\|X_{2}(s)\right\|^{2} d s, \\
& \alpha a_{0} b_{0}\left\langle X_{1}, \int_{t-\tau_{1}}^{t} B X_{3}(s) d s\right\rangle \leq \alpha a_{0} b_{0} b_{1}\left\|X_{1}\right\|\left\|\int_{t-\tau_{1}}^{t} X_{3}(s) d s\right\| \\
& \leq \alpha a_{0} b_{0} b_{1}\left\|X_{1}\right\| \int_{t-\tau_{1}}^{t}\left\|X_{3}(s)\right\| d s \\
& \leq \frac{1}{2} \alpha a_{0} b_{0} b_{1} \int_{t-\tau_{1}}^{t}\left\{\left\|X_{1}(t)\right\|^{2}+\left\|X_{3}(s)\right\|^{2}\right\} d s \\
& =\frac{1}{2} \alpha a_{0} b_{0} b_{1} \tau_{1}\left\|X_{1}\right\|^{2}+\frac{1}{2} \alpha a_{0} b_{0} b_{1} \int_{t-\tau_{1}}^{t}\left\|X_{3}(s)\right\|^{2} d s, \\
& \alpha a_{0} b_{0} c\left\langle X_{1}, \int_{t-\tau_{1}}^{t} X_{2}(s) d s\right\rangle \leq \alpha a_{0} b_{0} c\left\|X_{1}\right\|\left\|\int_{t-\tau_{1}}^{t} X_{2}(s) d s \mid\right\| \\
& \leq \alpha a_{0} b_{0} c\left\|X_{1}\right\| \int_{t-\tau_{1}}^{t}\left\|X_{2}(s)\right\| d s \\
& \leq \frac{1}{2} \alpha a_{0} b_{0} c \int_{t-\tau_{1}}^{t}\left\{\left\|X_{1}(t)\right\|^{2}+\left\|X_{2}(s)\right\|^{2}\right\} d s \\
& =\frac{1}{2} \alpha a_{0} b_{0} c \tau_{1}\left\|X_{1}\right\|^{2}+\frac{1}{2} \alpha a_{0} b_{0} c \int_{t-\tau_{1}}^{t}\left\|X_{2}(s)\right\|^{2} d s,
\end{aligned}
$$




$$
\begin{aligned}
& a_{0}\left\langle X_{2}, \int_{t-\tau_{1}}^{t} B X_{3}(s) d s\right\rangle \leq a_{0}\left\|X_{2}\right\|\left\|\int_{t-\tau_{1}}^{t} B X_{3}(s) d s\right\| \\
& \leq a_{0} b_{1}\left\|X_{2}\right\| \int_{t-\tau_{1}}^{t}\left\|X_{3}(s)\right\| d s \\
& \leq \frac{1}{2} a_{0} b_{1} \int_{t-\tau_{1}}^{t}\left\{\left\|X_{2}(t)\right\|^{2}+\left\|X_{3}(s)\right\|^{2}\right\} d s \\
& =\frac{1}{2} a_{0} b_{1} \tau_{1}\left\|X_{2}\right\|^{2}+\frac{1}{2} a_{0} b_{1} \int_{t-\tau_{1}}^{t}\left\|X_{3}(s)\right\|^{2} d s, \\
& a_{0} c\left\langle X_{2}, \int_{t-\tau_{1}}^{t} X_{2}(s) d s\right\rangle \leq a_{0} c\left\|X_{2}\right\| \int_{t-\tau_{1}}^{t} X_{2}(s) d s \| \\
& \leq a_{0} c\left\|X_{2}\right\| \int_{t-\tau_{1}}^{t}\left\|X_{2}(s)\right\| d s \\
& \leq \frac{1}{2} a_{0} c \int_{t-\tau_{1}}^{t}\left\{\left\|X_{2}(t)\right\|^{2}+\left\|X_{2}(s)\right\|^{2}\right\} d s \\
& =\frac{1}{2} a_{0} c \tau_{1}\left\|X_{2}\right\|^{2}+\frac{1}{2} a_{0} c \int_{t-\tau_{1}}^{t}\left\|X_{2}(s)\right\|^{2} d s .
\end{aligned}
$$

Hence, it follows that

$$
\begin{aligned}
& \dot{V}(t) \leq-\frac{1}{2} \alpha a_{0} b_{0} c\left\langle X_{1}, X_{1}\right\rangle-\left\langle\left(a_{0} B-c I-\alpha a_{0}^{2} b_{0} I\right) X_{2}, X_{2}\right\rangle \\
& -\left\langle\left(\Psi\left(X_{2}\right)-a_{0} I\right) X_{3}, X_{3}\right\rangle \\
& -\frac{1}{4} \alpha a_{0} b_{0}\left\|c^{\frac{1}{2}} X_{1}+2 c^{-\frac{1}{2}}\left(\Psi\left(X_{2}\right)-a_{0} I\right) X_{3}\right\|^{2} \\
& +\frac{1}{4} \alpha a_{0} b_{0}\left\|2 c^{-\frac{1}{2}}\left(\Psi\left(X_{2}\right)-a_{0} I\right) X_{3}\right\|^{2} \\
& -\frac{1}{4} \alpha a_{0} b_{0}\left\|c^{\frac{1}{2}} X_{1}+2 c^{-\frac{1}{2}}\left(B-b_{0} I\right) X_{2}\right\|^{2} \\
& +\frac{1}{4} \alpha a_{0} b_{0}\left\|2 c^{\frac{-1}{2}}\left(B-b_{0} I\right) X_{2}\right\|^{2} \\
& +\left.\frac{1}{2} \alpha a_{0} b_{0} b_{1} \tau_{1}\left\|X_{1}\right\|\right|^{2}+\frac{1}{2} \alpha a_{0} b_{0} c \tau_{1}\left\|X_{1}\right\|^{2} \\
& +\frac{1}{2} a_{0} b_{1} \tau_{1}\left\|X_{2}\right\|^{2}+\frac{1}{2} a_{0} c \tau_{1}\left\|X_{2}\right\|^{2} \\
& +\frac{1}{2} b_{1} \tau_{1}\left\|X_{3}\right\|^{2}+\frac{1}{2} c \tau_{1}\left\|X_{3}\right\|^{2} \\
& +\lambda_{1} \tau_{1}\left\langle X_{2}, X_{2}\right\rangle+\eta_{1} \tau_{1}\left\langle X_{3}, X_{3}\right\rangle
\end{aligned}
$$




$$
\begin{aligned}
& -\left\{\lambda_{1}-\frac{1}{2}\left(a_{0}+\alpha a_{0} b_{0}+1\right) c\right\} \int_{t-\tau_{1}}^{t}\left\|X_{2}(s)\right\|^{2} d s \\
& -\left\{\eta_{1}-\left(1+a_{0}+\frac{1}{2} \alpha a_{0} b_{0}\right) b_{1}\right\} \int_{t-\tau_{1}}^{t}\left\|X_{3}(s)\right\|^{2} d s .
\end{aligned}
$$

Let

$$
\lambda_{1}=\frac{1}{2}\left(a_{0}+\alpha a_{0} b_{0}+1\right) c
$$

and

$$
\eta_{1}=\left(1+a_{0}+\frac{1}{2} \alpha a_{0} b_{0}\right) b_{1} .
$$

Then

$$
\begin{aligned}
& \dot{V}(t) \leq-\frac{1}{2} \alpha a_{0} b_{0} c\left\langle X_{1}, X_{1}\right\rangle \\
& -\left\langle\left(a_{0} B-c I-\alpha a_{0}^{2} b_{0} I\right) X_{2}, X_{2}\right\rangle \\
& -\left\langle\left(\Psi\left(X_{2}\right)-a_{0} I\right) X_{3}, X_{3}\right\rangle \\
& +\frac{1}{4} \alpha a_{0} b_{0}\left\|2 c^{-\frac{1}{2}}\left(\Psi\left(X_{2}\right)-a_{0} I\right) X_{3}\right\|^{2} \\
& +\frac{1}{4} \alpha a_{0} b_{0}\left\|2 c^{-\frac{1}{2}}\left(B-b_{0} I\right) X_{2}\right\|^{2} \\
& +\frac{1}{2}\left(\alpha a_{0} b_{0} b_{1}+\alpha a_{0} b_{0} c\right) \tau_{1}\left\|X_{1}\right\|^{2}+\frac{1}{2}\left(a_{0} b_{1}+a_{0} c\right) \tau_{1}\left\|X_{2}\right\|^{2} \\
& +\frac{1}{2}\left(a_{0}+\alpha a_{0} b_{0}+1\right) c \tau_{1}\left\|X_{2}\right\|^{2}+\frac{1}{2}\left(b_{1}+c\right) \tau_{1}\left\|X_{3}\right\|^{2} \\
& +\frac{1}{2}\left(1+a_{0}+\alpha a_{0} b_{0}\right) b_{1} \tau_{1}\left\|X_{3}\right\|^{2} .
\end{aligned}
$$$$
\text { In view of the estimates }
$$$$
\frac{1}{4} \alpha a_{0} b_{0}\left\|2 c^{-\frac{1}{2}}\left(B-b_{0} I\right) X_{2}\right\|^{2}
$$$$
=\alpha a_{0} b_{0}\left\langle c^{-1}\left(B-b_{0} I\right) X_{2},\left(B-b_{0} I\right) X_{2}\right\rangle
$$

and

$$
\begin{aligned}
& \frac{1}{4} \alpha a_{0} b_{0}\left\|2 c^{-\frac{1}{2}}\left(\Psi\left(X_{2}\right)-a_{0} I\right) X_{3}\right\|^{2} \\
& =\alpha a_{0} b_{0}\left\langle\left(c^{-1}\left(\Psi\left(X_{2}\right)-a_{0} I\right)\right) X_{3},\left(\Psi\left(X_{2}\right)-a_{0} I\right) X_{3}>,\right.
\end{aligned}
$$

it follows that 


$$
\begin{aligned}
& \dot{V}(t) \leq-\frac{1}{2} \alpha a_{0} b_{0} c\left\langle X_{1}, X_{1}\right\rangle \\
& -\left\langle\left(a_{0} B-c I-\alpha a_{0}^{2} b_{0} I\right) X_{2}, X_{2}\right\rangle \\
& +\alpha a_{0} b_{0}\left\langle c^{-1}\left(B-b_{0} I\right) X_{2},\left(B-b_{0} I\right) X_{2}\right\rangle \\
& -\left\langle\left(\Psi\left(X_{2}\right)-a_{0} I\right) X_{3}, X_{3}\right\rangle \\
& +\alpha a_{0} b_{0}\left\langle c^{-1}\left(\Psi\left(X_{2}\right)-a_{0} I\right) X_{3},\left(\Psi\left(X_{2}\right)-a_{0} I\right) X_{3}\right\rangle \\
& +\frac{1}{2}\left(\alpha a_{0} b_{0} b_{1}+\alpha a_{0} b_{0} c\right) \tau_{1}\left\|X_{1}\right\|^{2}+\frac{1}{2}\left(a_{0} b_{1}+a_{0} c\right) \tau_{1}\left\|X_{2}\right\|^{2} \\
& +\frac{1}{2}\left(a_{0}+\alpha a_{0} b_{0}+1\right) c \tau_{1}\left\|X_{2}\right\|^{2}+\frac{1}{2}\left(b_{1}+c\right) \tau_{1}\left\|X_{3}\right\|^{2} \\
& +\frac{1}{2}\left(1+a_{0}+\alpha a_{0} b_{0}\right) b_{1} \tau_{1}\left\|X_{3}\right\|^{2} .
\end{aligned}
$$

By noting Lemma and the assumptions of Theorem 1, it can be obtained that

$$
\begin{aligned}
& \dot{V}(t) \leq-\frac{1}{2}\left\{\alpha a_{0} b_{0} c-\left(\alpha a_{0} b_{0} b_{1}+\alpha a_{0} b_{0} c\right) \tau_{1}\right\}\left\|X_{1}\right\|^{2} \\
& -\left\langle\left\{\left(a_{0} B-c I\right)-\alpha a_{0} b_{0}\left[a_{0} I+c^{-1}\left(B-b_{0} I\right)^{2}\right]\right\} X_{2}, X_{2}\right\rangle \\
& +\frac{1}{2}\left(a_{0} b_{1}+a_{0} c\right) \tau_{1}\left\|X_{2}\right\|^{2} \\
& +\frac{1}{2}\left(a_{0}+\alpha a_{0} b_{0}+1\right) c \tau_{1}\left\|X_{2}\right\|^{2} \\
& -\left\langle\left\{\left(\Psi\left(X_{2}\right)-a_{0} I\right)\left[I-\alpha a_{0} b_{0} c^{-1}\left(\Psi\left(X_{2}\right)-a_{0} I\right)\right]\right\} X_{3}, X_{3}\right\rangle \\
& +\frac{1}{2}\left(b_{1}+c\right) \tau_{1}\left\|X_{3}\right\|^{2} \\
& +\frac{1}{2}\left(1+a_{0}+\alpha a_{0} b_{0}\right) b_{1} \tau_{1}\left\|X_{3}\right\|^{2} \\
& \leq-\frac{1}{2}\left\{\alpha a_{0} b_{0} c-\left(\alpha a_{0} b_{0} b_{1}+\alpha a_{0} b_{0} c\right) \tau_{1}\right\}\left\|X_{1}\right\|^{2} \\
& -\left\{\left(a_{0} b_{0}-c\right)-\alpha a_{0} b_{0}\left[a_{0}+c^{-1}\left(b_{1}-b_{0}\right)^{2}\right]\right\}\left\|X_{2}\right\|^{2} \\
& +\frac{1}{2}\left\{\left(2 a_{0}+\alpha a_{0} b_{0}+1\right) c+a_{0} b_{1}\right\} \tau_{1}\left\|X_{2}\right\|^{2} \\
& -\varepsilon\left[1-\alpha a_{0} b_{0} c^{-1}\left(a_{1}-a_{0}\right)^{2}\right]\left\|X_{3}\right\|^{2}
\end{aligned}
$$


$+\frac{1}{2}\left(2 b_{1}+c+a_{0} b_{1}+\alpha a_{0} b_{0} b_{1}\right) \tau_{1}\left\|X_{3}\right\|^{2}$.

Let

$$
k_{1}=2\left(a_{0} b_{0}-c\right)-\alpha a_{0} b_{0}\left[a_{0}+c^{-1}\left(b_{1}-b_{0}\right)^{2}\right]>0
$$

and

$$
k_{2}=2 \varepsilon\left[1-\alpha a_{0} b_{0} c^{-1}\left(a_{1}-a_{0}\right)^{2}\right]>0 .
$$

Hence

$$
\begin{aligned}
& \dot{V}(t) \leq-\frac{1}{2}\left\{\alpha a_{0} b_{0} c-\left(\alpha a_{0} b_{0} b_{1}+\alpha a_{0} b_{0} c\right) \tau_{1}\right\}\left\|X_{1}\right\|^{2} \\
& -\frac{1}{2}\left\{k_{1}-\left[\left(2 a_{0}+\alpha a_{0} b_{0}+1\right) c+a_{0} b_{1}\right] \tau_{1}\right\}\left\|X_{2}\right\|^{2} \\
& -\frac{1}{2}\left\{k_{2}-\left(2 b_{1}+c+a_{0} b_{1}+\alpha a_{0} b_{0} b_{1}\right) \tau_{1}\right\}\left\|X_{3}\right\|^{2} .
\end{aligned}
$$

If

$$
\begin{gathered}
\tau_{1}<\min \left\{\frac{\alpha a_{0} b_{0} c}{\alpha a_{0} b_{0} b_{1}+\alpha a_{0} b_{0} c}, \frac{k_{1}}{\left(2 a_{0}+\alpha a_{0} b_{0}+1\right) c+a_{0} b_{1}},\right. \\
\left.\frac{k_{2}}{c+\left(2+a_{0}+\alpha a_{0} b_{0}\right) b_{1}}\right\},
\end{gathered}
$$

then, for some positive constants $\rho_{1}, \rho_{2}$ and $\rho_{3}$, it follows that

$$
\dot{V}(t) \leq-\rho_{1}\left\|X_{1}\right\|^{2}-\rho_{2}\left\|X_{2}\right\|^{2}-\rho_{3}\left\|X_{3}\right\|^{2} \leq 0 .
$$

In addition, we can easily see that

$$
V\left(X_{1}, X_{2}, X_{3}\right) \rightarrow \infty \text { as }\left\|X_{1}\right\|^{2}+\left\|X_{2}\right\|^{2}+\left\|X_{3}\right\|^{2} \rightarrow \infty .
$$

We will now apply LaSalle's invariance principle, so consider the set

$$
E \equiv\left\{\left(X_{1}, X_{2}, X_{3}\right): \dot{V}\left(X_{1}, X_{2}, X_{3}\right)=0\right\} .
$$

Observe that $\left(X_{1}, X_{2}, X_{3}\right) \in E$ implies that $X_{1}=X_{2}=X_{3}=0$. Clearly, the largest invariant set contained in $E$ is $(0,0,0) \in E$, and so the zero solution of system (2) is globally asymptotically stable. Hence, the zero solution of Eq. (1) is globally asymptotically stable.

This completes the proof of Theorem 1 . 
In the case $P(t) \neq 0$, the second result of this paper is the following theorem.

Theorem 2. We assume that all assumptions of Theorem 1 hold, except $P(t) \equiv 0$ In addition, we assume that there exists a non-negative and continuous function $\theta=\theta(t)$ such that the following condition holds:

$$
\|P(t)\| \leq \theta(t) \text { for all } t \geq 0, \max \theta(t)<\infty \text { and } \theta \in L^{1}(0, \infty),
$$

where $L^{1}(0, \infty)$ denotes the space of Lebesgue integrable functions.

If

$$
\begin{gathered}
\tau_{1}<\min \left\{\frac{\alpha a_{0} b_{0} c}{\alpha a_{0} b_{0} b_{1}+\alpha a_{0} b_{0} c},\right. \\
\left.\frac{k_{1}}{\left(2 a_{0}+\alpha a_{0} b_{0}+1\right) c+a_{0} b_{1}}, \frac{k_{2}}{c+\left(2+a_{0}+\alpha a_{0} b_{0}\right) b_{1}}\right\},
\end{gathered}
$$

where

$$
k_{1}=2\left(a_{0} b_{0}-c\right)-\alpha a_{0} b_{0}\left[a_{0}+c^{-1}\left(b_{1}-b_{0}\right)^{2}\right]>0
$$

and

$$
k_{2}=2 \varepsilon\left[1-\alpha a_{0} b_{0} c^{-1}\left(a_{1}-a_{0}\right)^{2}\right]>0,
$$

then there exists a constant $D>0$ such that any solution $\left(X_{1}(t), X_{2}(t), X_{3}(t)\right)$ of system (2) determined by

$$
X_{1}(0)=X_{10}, X_{2}(0)=X_{20}, X_{3}(0)=X_{30}
$$

satisfies

$$
\left\|X_{1}(t)\right\| \leq D,\left\|X_{2}(t)\right\| \leq D,\left\|X_{3}(t)\right\| \leq D
$$

for all $t \in \Re^{+}$.

Proof. In the case of $P(t) \neq 0$ under the assumptions of Theorem 2 , it can be easily seen that

$$
\begin{aligned}
& \dot{V}(t) \leq-\frac{1}{2}\left\{\alpha a_{0} b_{0} c-\left(\alpha a_{0} b_{0} b_{1}+\alpha a_{0} b_{0} c\right) \tau_{1}\right\}\left\|X_{1}\right\|^{2} \\
& -\frac{1}{2}\left\{k_{1}-\left[\left(2 a_{0}+\alpha a_{0} b_{0}+1\right) c+a_{0} b_{1}\right] \tau_{1}\right\}\left\|X_{2}\right\|^{2}
\end{aligned}
$$




$$
\begin{aligned}
& -\frac{1}{2}\left\{k_{2}-\left(2 b_{1}+c+a_{0} b_{1}+\alpha a_{0} b_{0} b_{1}\right) \tau_{1}\right\}\left\|X_{3}\right\|^{2} \\
& +\left\langle X_{3}, P(t)\right\rangle+\alpha a_{0} b_{0}\left\langle X_{1}, P(t)\right\rangle+a_{0}\left\langle X_{2}, P(t)\right\rangle \\
& \leq\left(\alpha a_{0} b_{0}\left\|X_{1}\right\|+a_{0}\left\|X_{2}\right\|+\left\|X_{3}\right\|\right)\|P(t)\| \\
& \leq \sigma\left(\left\|X_{1}\right\|+\left\|X_{2}\right\|+\left\|X_{3}\right\|\right)\|P(t)\| \\
& \leq \sigma\left(3+\left\|X_{1}\right\|^{2}+\left\|X_{2}\right\|^{2}+\left\|X_{3}\right\|^{2}\right) \theta(t),
\end{aligned}
$$

where

$$
\sigma=\max \left\{\alpha a_{0} b_{0}, a_{0}, 1\right\} .
$$

On the other hand, it is easy to see that

$$
\left\|X_{1}\right\|^{2}+\left\|X_{2}\right\|^{2}+\left\|X_{3}\right\|^{2} \leq d_{4}^{-1} V .
$$

In view of the above discussion, we get

$$
\dot{V}(t) \leq 3 \sigma \theta(t)+d_{4}^{-1} V(t) \theta(t) .
$$

Integrating both sides of the last estimate from 0 to $t,(t \geq 0)$, one can easily obtain

$$
V(t)-V(0) \leq 3 \sigma \int_{0}^{t} \theta(s) d s+d_{4}^{-1} \int_{0}^{t} V(s) \theta(s) d s .
$$

Then

$$
V(t) \leq d+d_{4}^{-1} \int_{0}^{\infty} V(s) \theta(s) d s
$$

where

$$
d=V(0)+3 \sigma \int_{0}^{\infty} \theta(s) d s .
$$

By using Gronwall-Bellman inequality (see Ahmad and Rama Mohana Rao [4]), we conclude that

$$
V(t) \leq d \exp \left(d_{4}^{-1} \int_{0}^{\infty} \theta(s)\right) d s .
$$


Thus, all solutions of system (2) are bounded.

For the case $P(t) \neq 0$, the third and last main result is the following theorem.

Theorem 3. We assume that all assumptions of Theorem 1 hold, except $P(t) \equiv 0$. In addition, we assume that there exists a positive constant $\delta_{0}$ such that the condition

$$
\|P(t)\| \leq \delta_{0},(t \geq 0)
$$

holds.

If

$$
\begin{gathered}
\tau_{1}<\min \left\{\frac{\alpha a_{0} b_{0} c}{\alpha a_{0} b_{0} b_{1}+\alpha a_{0} b_{0} c},\right. \\
\left.\frac{k_{1}}{\left(2 a_{0}+\alpha a_{0} b_{0}+1\right) c+a_{0} b_{1}}, \frac{k_{2}}{c+\left(2+a_{0}+\alpha a_{0} b_{0}\right) b_{1}}\right\},
\end{gathered}
$$

where

$$
k_{1}=2\left(a_{0} b_{0}-c\right)-\alpha a_{0} b_{0}\left[a_{0}+c^{-1}\left(b_{1}-b_{0}\right)^{2}\right]>0
$$

and

$$
k_{2}=2 \varepsilon\left[1-\alpha a_{0} b_{0} c^{-1}\left(a_{1}-a_{0}\right)^{2}\right]>0,
$$

then there exists a constant $d>0$ such that any solution $\left(X_{1}(t), X_{2}(t), X_{3}(t)\right)$ of system (2) determined by

$$
X_{1}(0)=X_{10}, X_{2}(0)=X_{20}, X_{3}(0)=X_{30}
$$

ultimately satisfies

$$
\left\|X_{1}(t)\right\|^{2}+\left\|X_{2}(t)\right\|^{2}+\left\|X_{3}(t)\right\|^{2} \leq d
$$

for all $t \in \Re^{+}$.

Proof. When $P(t) \neq 0$, under the assumptions of Theorem 3, we can re-arrange the time derivative of the Liapunov- Krasovskii functional $\dot{V}(t)$ as the following:

$$
\dot{V}(t) \leq-\rho_{1}\left\|X_{1}\right\|^{2}-\rho_{2}\left\|X_{2}\right\|^{2}-\rho_{3}\left\|X_{3}\right\|^{2}
$$




$$
\begin{aligned}
& +\left(\alpha a_{0} b_{0}\left\|X_{1}\right\|^{2}+a_{0}\left\|X_{2}\right\|+\left\|X_{3}\right\|\right)\|P(t)\| \\
& \leq-\rho_{1}\left\|X_{1}\right\|^{2}-\rho_{2}\left\|X_{2}\right\|^{2}-\rho_{3}\left\|X_{3}\right\|^{2} \\
& +\left(\alpha a_{0} b_{0} \delta_{0}\left\|X_{1}\right\|^{2}+a_{0} \delta_{0}\left\|X_{2}\right\|+\delta_{0}\left\|X_{3}\right\|\right) .
\end{aligned}
$$

Let

$$
\bar{d}_{2}=\frac{1}{2} \max \left\{\rho_{1}, \rho_{2}, \rho_{3}\right\}
$$

and

$$
\bar{d}_{3}=\max \left\{\alpha a_{0} b_{0} \delta_{0}, a_{0} b_{0} \delta_{0}, \delta_{0}\right\}
$$

Therefore,

$$
\dot{V}(t) \leq-2 \bar{d}_{2}\left\{\left\|X_{1}\right\|^{2}+\left\|X_{2}\right\|^{2}+\left\|X_{3}\right\|^{2}\right\}+\bar{d}_{3}\left(\left\|X_{1}\right\|+\left\|X_{2}\right\|+\left\|X_{3}\right\|\right) .
$$

In view of Schwarz's inequality, it can be written that

$$
\dot{V}(t) \leq-2 \bar{d}_{2}\left\{\left\|X_{1}\right\|^{2}+\left\|X_{2}\right\|^{2}+\left\|X_{3}\right\|^{2}\right\}+\bar{d}_{4}\left(\left\|X_{1}\right\|^{2}+\left\|X_{2}\right\|^{2}+\left\|X_{3}\right\|^{2}\right)^{\frac{1}{2}}
$$

where $\bar{d}_{4}=\sqrt{3} \bar{d}_{3}$.

If

$$
\left(\left\|X_{1}\right\|^{2}+\left\|X_{2}\right\|^{2}+\left\|X_{3}\right\|^{2}\right)^{\frac{1}{2}} \geq \bar{d}_{5}=\bar{d}_{4} \bar{d}_{2}^{-1},
$$

then we get

$$
\dot{V}(t) \leq-\bar{d}_{2}\left(\left\|X_{1}\right\|^{2}+\left\|X_{2}\right\|^{2}+\left\|X_{3}\right\|^{2}\right) .
$$

Hence, it follows that there exists a positive constants $\bar{d}_{6}$ such that

$$
\dot{V}(t) \leq-1
$$

if ||$X_{1}\left\|^{2}+\right\| X_{2}\left\|^{2}+\right\| X_{3} \|^{2} \geq d_{6}^{2}$.

The remaining of the proof can be completed easily by following a similar was as shown in Meng [21]. Therefore, we would not like to give the details of the proof.

\section{Conclusion}

A class of vector functional differential equations of third order with a constant retardation has been considered. The stability/boundedness/ ultimately boundedness of solutions of these equations have been discussed by using the Liapunov- Krasovskii functional approach. The obtained results extend and improve some recent results in the literature. 


\section{References}

[1] T. A. Ademola; P. O. Arawomo, Asymptotic behaviour of solutions of third order nonlinear differential equations. Acta Univ. Sapientiae Math. 3, No. 2, pp. 197-211, (2011).

[2] A. U. Afuwape; J. E. Castellanos, Asymptotic and exponential stability of certain third-order non-linear delayed differential equations: frequency domain method. Appl. Math. Comput. 216, No. 3, pp. 940950, (2010).

[3] A. U. Afuwape; P. Omari; F. Zanolin, Nonlinear perturbations of differential operators with nontrivial kernel and applications to thirdorder periodic boundary value problems. J. Math. Anal. Appl. 143, No. 1, pp. 35-56, (1989).

[4] S. Ahmad; M. Rama Mohana Rao, Theory of ordinary differential equations. With applications in biology and engineering. Affiliated East-West Press Pvt. Ltd., New Delhi, (1999).

[5] T. A. Burton, Stability and periodic solutions of ordinary and functional differential equations. Academic Press, Orlando, (1985).

[6] K. E. Chlouverakis; J. C. Sprott, Chaotic hyperjerk systems. Chaos Solitons Fractals 28, No. 3, pp. 739-746, (2006).

[7] E. N. Chukwu, On boundedness of solutions of third order differential equations. Ann. Math. Pura. Appl. 104, pp. 123-149, (1975).

[8] J. Cronin-Scanlon, Some mathematics of biological oscillations. SIAM Rev. 19, No. 1, pp. 100-138, (1977).

[9] Z. Elhadj; J. C. Sprott, Boundedness of certain forms of jerky dynamics. Qual. Theory Dyn. Syst. 11, No. 2, pp. 199-213, (2012).

[10] R. Eichhorn; S. J. Linz; P. HÃnggi, Transformations of nonlinear dynamical systems to jerky motion and its application to minimal chaotic flows. Phys Rev E 58, pp. 7151-7164, (1998). 
[11] Èl'sgol'ts, L. E.; Norkin, S. B., Introduction to the theory and application of differential equations with deviating arguments. Translated from the Russian by John L. Casti. Mathematics in Science and Engineering, Vol. 105. Academic Press, New York-London, (1973).

[12] J. O. C. Ezeilo, On the stability of solutions of certain differential equations of the third order. Quart. J. Math. Oxford Ser. (2) 11, pp. 64-69, (1960).

[13] Ezeilo, J. O. C., A generalization of a boundedness theorem for a certain third-order differential equation. Proc. Cambridge Philos. Soc. 63, pp. 735-742, (1967).

[14] K. O. Fridedrichs, On nonlinear vibrations of third order. Studies in Nonlinear Vibration Theory, pp. 65-103. Institute for Mathematics and Mechanics, New York University, (1946).

[15] J. Hale, Sufficient conditions for stability and instability of autonomous functional-differential equations. J. Differential Equations 1, pp. 452482, (1965).

[16] T. Hara, On the uniform ultimate boundedness of the solutions of certain third order differential equations. J. Math. Anal. Appl. 80, No. 2, pp. 533-544, (1981).

[17] N. N. Krasovskií, Stability of Motion. Applications of Lyapunov's Second Method to Differential Systems and Equations with Delay, Stanford, Calif.: Stanford University Press (1963).

[18] A. M. Liapunov, Stability of motion. With a contribution by V. A. Pliss and an introduction by V. P. Basov. Translated from the Russian by Flavian Abramovici and Michael Shimshoni. Mathematics in Science and Engineering, Vol. 30 Academic Press, New York-London (1966).

[19] S. J. Linz, On hyperjerky systems. Chaos Solitons Fractals 37, No. 3, pp. $741-747,(2008)$.

[20] B. Mehri; D. Shadman, Boundedness of solutions of certain third order differential equation. Math. Inequal. Appl. 2, No.4, pp. 545-549, (1999).

[21] F. W. Meng, Ultimate boundedness results for a certain system of third order nonlinear differential equations. J. Math. Anal. Appl. 177, No. 2, pp. 496-509, (1993). 
[22] M. O.Omeike; A. U. Afuwape, New result on the ultimate boundedness of solutions of certain thir d-order vector differential equations. Acta Univ. Palack. Olomuc. Fac. Rerum Natur. Math. 49, No. 1, pp. 55-61, (2010).

[23] C. Qian, On global stability of third-order nonlinear differential equations. Nonlinear Anal. 42, No. 4, Ser. A: Theory Methods, pp. 651-661, (2000).

[24] L. L. Rauch, Oscillation of a third order nonlinear autonomous system. Contributions to the Theory of Nonlinear Oscillations, pp. 39-88. Annals of Mathematics Studies, no. 20. Princeton University Press, Princeton, N.J., (1950).

[25] R. Reissig; G. Sansone; R. Conti, Non-linear differential equations of higher order. Translated from the German. Noordhoff International Publishing, Leyden, (1974).

[26] H. Smith, An introduction to delay differential equations with applications to the life sciences. Texts in Applied Mathematics, 57. Springer, New York, (2011).

[27] K. E. Swick, Asymptotic behavior of the solutions of certain third order differential equations. SIAM J. Appl. Math. 19, pp. 96-102, (1970).

[28] H. O. Tejumola, A note on the boundedness and the stability of solutions of certain third-order differential equations. Ann. Mat. Pura Appl. (4) 92, pp. 65-75, (1972).

[29] C. Tunç, Global stability of solutions of certain third-order nonlinear differential equations. Panamer. Math. J. 14, No. 4, pp. 31-35, (2004).

[30] C. Tunç, Uniform ultimate boundedness of the solutions of third-order nonlinear differential equations. Kuwait J. Sci. Engrg. 32, No. 1, pp. 39-48, (2005).

[31] C. Tunç, Boundedness of solutions of a third-order nonlinear differential equation. JIPAM. J. Inequal. Pure Appl. Math. 6, No. 1, Article 3,6 pp. - , (2005)

[32] C. Tunç, On the asymptotic behavior of solutions of certain third-order nonlinear differential equations. J. Appl. Math. Stoch. Anal., No. 1, pp. 29-35, (2005). 
[33] C. Tunç, Some instability results on certain third order nonlinear vector differential equations. Bull. Inst. Math. Acad. Sin. (N.S.) 2 (2007), no. $1,109-122$.

[34] C. Tunç, On the stability and boundedness of solutions to third order nonlinear differential equations with retarded argument. Nonlinear Dynam. 57, No. 1-2, pp. 97-106, (2009).

[35] C. Tunç, On the stability and boundedness of solutions of nonlinear vector differential equations of third order. Nonlinear Anal. 70, No. 6, pp. 2232-2236, (2009).

[36] C. Tunç, Stability and bounded of solutions to non-autonomous delay differential equations of third order. Nonlinear Dynam. 62 (2010), No. 4, pp. 945-953, (2010).

[37] C. Tunç, On some qualitative behaviors of solutions to a kind of third order nonlinear delay differential equations. Electron. J. Qual. Theory Differ. Equ., No. 12, 19, pp. -, (2010).

[38] C. Tunç, Stability and boundedness for a kind of non-autonomous differential equations with constant delay. Appl. Math. Inf. Sci. 7, No. 1, pp. 355-361, (2013).

[39] C. Tunç, Stability and boundedness of the nonlinear differential equations of third order with multiple deviating arguments. Afr. Mat. 24, No. 3, pp. 381-390, (2013).

[40] C. Tunç, On the qualitative behaviors of solutions of some differential equations of higher order with multiple deviating arguments. J. Franklin Inst. 351, No. 2, pp. 643-655, (2014).

[41] C. Tunç; M. Ates, Stability and boundedness results for solutions of certain third order nonlinear vector differential equations. Nonlinear Dynam. 45, No. 3-4, pp. 273-281, (2006).

[42] C. Tunc; H. Ergören, Uniformly boundedness of a class of non-linear differential equations of third order with multiple deviating arguments. Cubo 14, No. 3, 63-69, (2012).

[43] E. T. Wall; M. L. Moe, An energy metric algorithm for the generation of Liapunov functions. IEEE Trans. Automat. Control. 13 (1), pp. 121-122, (1968). 
[44] L. Zhang; L. Yu, Global asymptotic stability of certain third-order nonlinear differential equations. Math. Methods Appl. Sci. Math. Methods Appl. Sci. 36, No. 14, pp. 1845-1850, (2013).

\author{
Cemil Tunç \\ Department of Mathematics, \\ Faculty of Sciences, \\ Yüzüncü Yil University, \\ 65080 Van, \\ Turkey \\ e-mail : cemtunc@yahoo.com \\ and
}

\title{
Sizar Abid Mohammed
}

University of Duhok,

Zakko Street 38,

1006 AJ Duhok,

Iraq

e-mail : sizar@uod.ac 